\title{
Two-Step Integral Imaging Coding Based Three-Dimensional Information Encryption Approach
}

\author{
Min Zhao $(\mathbb{D}$, Yan Xing, Xiao-Wei Li, and Qiong-Hua Wang $\mathbb{B}$ \\ School of Electronics and Information Engineering, Sichuan University, Chengdu 610065, China \\ Correspondence should be addressed to Qiong-Hua Wang; qhwang@scu.edu.cn
}

Received 26 October 2017; Accepted 14 November 2017; Published 16 January 2018

Academic Editor: Muhammad R. Abuturab

Copyright (C) 2018 Min Zhao et al. This is an open access article distributed under the Creative Commons Attribution License, which permits unrestricted use, distribution, and reproduction in any medium, provided the original work is properly cited.

\begin{abstract}
We propose a two-step integral imaging coding based three-dimensional (3D) information encryption approach in this paper. In this approach, a synthetic aperture integral imaging system is applied to acquire a set of parallax images including spatial and angular information of 3D scene. In the encryption process, two-step coding is performed. In the first step, the acquired parallax images are encrypted firstly by double random-phase coding in the Fresnel domain. In the second step, these encrypted parallax images are encoded into a cipher image by mapping algorithm which is used to generate elemental image array of integral imaging. In the decryption process, an inverse operation is performed. The experimental results demonstrate the feasibility, security, and robustness of the proposed approach.
\end{abstract}

\section{Introduction}

For the past decade, the autostereoscopic techniques without any auxiliary devices have gained more attention. Integral imaging (II) is considered as one of the attractive threedimensional (3D) technologies since it can provide both horizontal and vertical parallaxes and quasi-continuous viewing angles [1-4]. II consists of pickup process and reconstruction process. In the pickup process, the conventional II system inserts a microlens array in front of the image sensor to capture multiple images of a 3D scene from different perspectives $[3,5]$. In this way, 3D information is limited because the resolution of elemental image is restricted by image sensor resolution and microlens size, and the aberrations and diffraction also degrade the image quality $[6,7]$. To overcome these disadvantages, II pickup process can be implemented by using a camera array which can capture a larger field of view and high-resolution parallax images [8]. A synthetic aperture integral imaging (SAII) system in which a single camera shifts on a $2 \mathrm{D}$ plane to capture different perspective information can take the place of the camera array to reduce cost and the complexity of devices [9]. In the reconstruction process, optical integral imaging reconstruction (OIIR) and computational integral imaging reconstruction (CIIR) can be implemented to reconstruct the 3D information [10-12]. With the rapid development of 3D technologies, the 3D information encryption has become an urgent problem due to 3D information transmission security.

Optical encryption methods have attached much attention because they have various merits [13-25]. Double random-phase encoding in the Fresnel domain was proposed in 2004, and the encryption method is more flexible and compact because it is lensless and has higher security [26]. In recent years, some image encryption methods have also been proposed based on II [27-29]. An image encryption method using II and pixel scrambling technique was proposed [30]. In this method, the image is scrambled firstly by pixel scrambling technique; then the scrambled image is picked up through a microlens array. Finally, the acquired elemental image array (EIA) is scrambled again to obtain encrypted image. However, the method is only applied to encrypt a $2 \mathrm{D}$ image. $3 \mathrm{D}$ image encryption techniques based on computational integral imaging were proposed [31]. In the method, computational integral imaging is applied to pick up double images to generate EIA; then the EIA is encrypted by a cryptographic technology. However, the technique cannot be applied to the real 3D scene. A 3D information encryption system based on II and multiple 


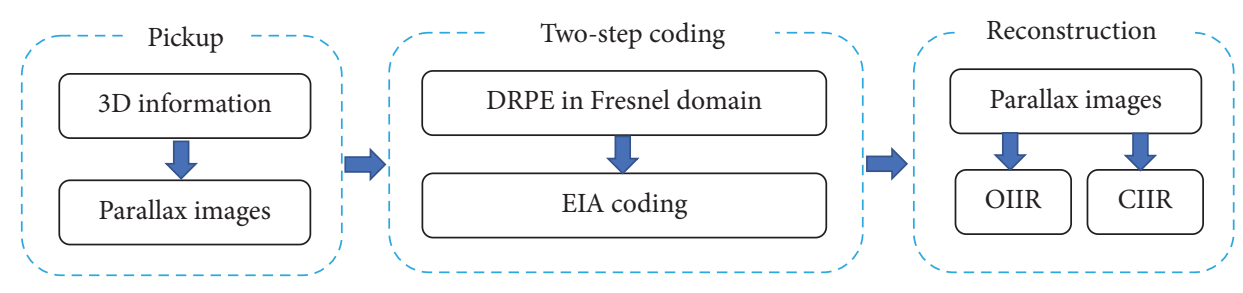

FIGURE 1: Schematic of the proposed encryption approach.

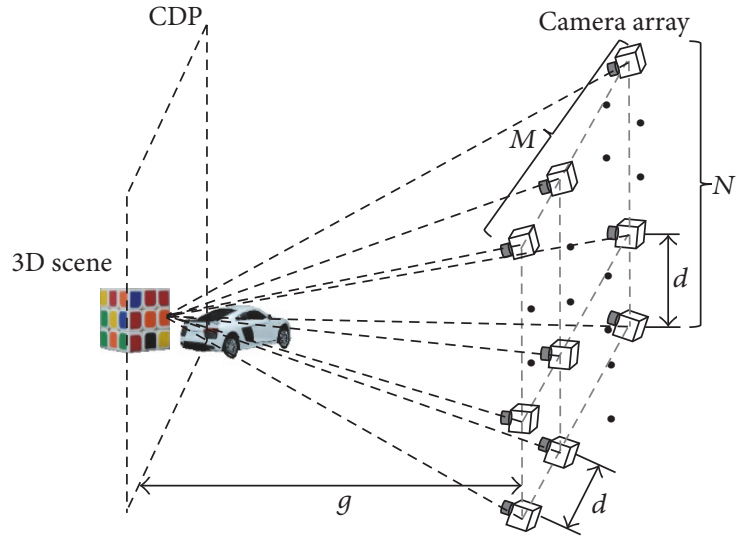

FIGURE 2: Schematic of the SAII system.

chaotic maps was proposed [32]. The system uses three chaotic maps to encrypt, respectively, three channels of EIA acquired by II. The encryption system is applied to real $3 \mathrm{D}$ scene.

In this paper, we propose a two-step coding 3D information encryption approach which is applied to real 3D scene based on an SAII system and double random-phase encoding (DRPE) in Fresnel domain, which improves further security and robustness. Meanwhile, the approach can reconstruct the flexible $3 \mathrm{D}$ information according to the II system configuration. In the process of two-step coding, parallax images encrypted by DRPE in Fresnel domain are interweaved to generate the cipher image using the mapping algorithm. In addition, the robustness of the proposed method is very high due to the data redundancy property of EIA. The experimental results verify the security and robustness of the proposed encryption approach, and the $3 \mathrm{D}$ information can be reconstructed flexibly by OIIR and CIIR according to II system configuration.

\section{Theoretical Analysis}

The proposed 3D information encryption approach is illustrated in Figure 1, and it consists of three parts: pickup, twostep coding, and reconstruction. In the pickup part, the $3 \mathrm{D}$ information is acquired firstly by using a SAII system, and $M \times N$ parallax images which record different perspectives of $3 \mathrm{D}$ scene can be obtained. In the two-step coding part, the DRPE in Fresnel domain is applied to encrypt parallax images with different secret keys. Then a mapping algorithm

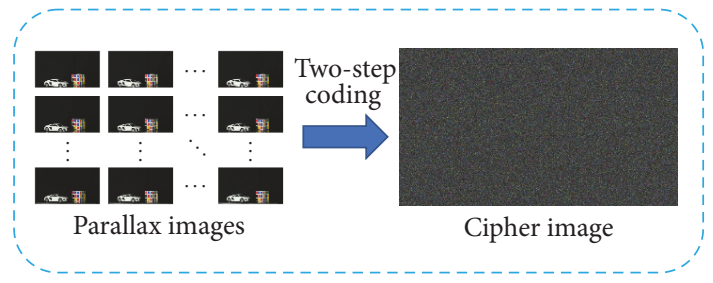

FIGURE 3: Schematic of the 3D information encryption.

is applied to interweave the encrypted parallax images to generate the cipher image. In the $3 \mathrm{D}$ information reconstruction part, the cipher image is divided into $M \times N$ images by using the inverse process of the EIA mapping algorithm. Then the decryption algorithm is applied to revive parallax images, and the $3 \mathrm{D}$ information is reconstructed flexibly by OIIR or CIIR.

2.1. Acquisition of 3D Information Using SAII System. In this part, the $3 \mathrm{D}$ information is acquired by an SAII system, in which a single camera is shifted on a $2 \mathrm{D}$ plane to capture different perspectives of the 3D scene. As shown in Figure 2, the SAII system can be considered as a camera array which is composed of $M \times N$ cameras. These cameras are located at the same plane, and the distance between adjacent cameras is $d$. The optical axes of the cameras converge to point $O$ which is located at the center of the central depth plane (CDP). Based on this SAII system, a set of parallax images recording $3 \mathrm{D}$ information of the scene are acquired.

2.2. Analysis of the Two-Step Coding. The proposed encryption approach performs two-step coding to improve the security and robustness. The schematic of the encryption process is shown in Figure 3. In the first step, we use DRPE in Fresnel domain to encrypt recorded parallax images. The locations of the first random-phase mask $\mathrm{RPM}_{m, n}^{1}$, the second random-phase mask $\mathrm{RPM}_{m, n}^{2}$, and the encrypted image are defined as the input plane, the relay plane, and the output plane, respectively. For convenience, we define the $(m, n)$ th parallax image and its corresponding encrypted image to be $f_{m, n}(x, y)$ and $\xi_{m, n}\left(x^{\prime \prime}, y^{\prime \prime}\right)$, respectively. $\exp [j \psi(x, y)]$ and $\exp \left[j \phi\left(x^{\prime}, y^{\prime}\right)\right]$ are used to represent $\mathrm{RPM}_{m, n}^{1}$ and $\mathrm{RPM}_{m, n}^{2}$. The parallax image is located on the position which is attached to the input plane. A plane wave with $\lambda$ wavelength is used to illuminate the input image perpendicularly. The parallax image $f_{m, n}(x, y)$ is modified by $\mathrm{RPM}_{m, n}^{1}$, then travelling the distance $z_{m, n}^{1}$ to relay plane. In the case of satisfying 
Fresnel approximation, complex amplitude $\mu_{m, n}\left(x^{\prime}, y^{\prime}\right)$ in the relay plane can be expressed as

$$
\begin{aligned}
\mu_{m, n}\left(x^{\prime}, y^{\prime}\right)= & \operatorname{FT}\left\{f_{m, n}(x, y) \exp [j \psi(x, y)]\right\} \\
& \times \exp \left[\frac{j \pi}{\lambda z_{m, n}^{1}} \times\left(\hat{x}^{2}+\hat{y}^{2}\right)\right],
\end{aligned}
$$

where $\hat{x}$ and $\hat{y}$ represent the spatial frequencies and FT represents Fourier transform. For convenience, we assume

$$
T_{\lambda z_{m, n}^{n}}\{\cdot\}=\mathrm{FT}\{\cdot\} \times \exp \left[\frac{j \pi}{\lambda z_{m, n}^{n}} \times\left[\hat{x}^{2}+\hat{y}^{2}\right]\right] .
$$

Then $\mu_{m, n}\left(x^{\prime}, y^{\prime}\right)$ is modified by $\mathrm{RPM}_{m, n}^{2}$, then travelling the distance $z_{m, n}^{2}$ to the output plane to obtain the encrypted parallax image, and the complex amplitude $\xi_{m, n}\left(x^{\prime \prime}, y^{\prime \prime}\right)$ in the output plane can be expressed as follows:

$$
\begin{aligned}
\xi_{m, n} & \left(x^{\prime \prime}, y^{\prime \prime}\right) \\
& =T_{\lambda z_{m, n}^{2}}\left\{T_{\lambda z_{m, n}^{1}}\left\{f_{m, n}(x, y) \exp [j \psi(x, y)]\right\}\right. \\
& \left.\times \exp \left[j \phi\left(x^{\prime}, y^{\prime}\right)\right]\right\} .
\end{aligned}
$$

After acquiring $M \times N$ encrypted parallax images, the secondstep coding is performed. A mapping algorithm which generates EIA is applied to interweave the encrypted parallax images to generate the cipher image. The cipher image $C(x, y)$ can be denoted as

$$
\begin{aligned}
C(x, y)=\left(\frac{p}{\Delta r}\right)^{2} \sum_{m, n} \sum_{i, j} \xi_{m, n}\left(\frac{p \cdot i}{\Delta r}-m\right. & \left.+V, \frac{p \cdot j}{\Delta r}-n+V\right) \cdot \delta\left(x-\frac{p \cdot i}{\Delta r}+m-V, y-\frac{p \cdot j}{\Delta r}+n-V\right), \\
& i=0,1, \ldots, \text { floor }\left(W \cdot \frac{\Delta r}{p}\right)-1, j=0,1, \ldots, \text { floor }\left(H \cdot \frac{\Delta r}{p}\right)-1, V=\frac{(p+\Delta r)}{\Delta r},
\end{aligned}
$$

where $\Delta r$ is the pixel size of the encrypted parallax images, $p$ represents the pitch of the virtual microlens, and $W \times H$ is the resolution of the parallax images.

The proposed encryption approach has multiple group secret keys, and we can adopt different secret keys or the same secret keys to encrypt each parallax image. And, the parameters of the mapping algorithm can be considered as a part of secret keys. Therefore, the approach has great security.

2.3. Analysis of $3 D$ Information Reconstruction. In decryption process, at first, the cipher image is divided into $M \times N$ images by using inverse EIA mapping algorithm. Then, the encrypted parallax images are decoded through the following operation:

$$
\begin{aligned}
& f_{m, n}(x, y) \\
& \quad=T_{\lambda z_{m, n}^{2}}\left\{T_{\lambda z_{m, n}^{1}}\left\{\xi_{m, n}\left(x^{\prime \prime}, y^{\prime \prime}\right) \exp [-j \psi(x, y)]\right\}\right. \\
& \left.\quad \times \exp \left[-j \phi\left(x^{\prime}, y^{\prime}\right)\right]\right\} .
\end{aligned}
$$

The $M \times N$ parallax images are decrypted by corresponding secret keys, respectively. The 3D information can be recovered by II reconstruction which is performed by two methods: OIIR and CIIR. In the OIIR, 3D information is recovered according to the principle of reversibility of the light rays, which is the inverse of the pickup stage. In the CIIR technique, according to the geometric optics, 3D information can be constructed through mapping inversely parallax images into object space, as shown in Figure 4. The reconstructed image located the depth of $z$ can be expressed as follows:

$$
R(x, y, z)=\frac{1}{N(x, y)}
$$

$$
\cdot \sum_{m=0}^{M-1} \sum_{n=0}^{N-1} f_{m, n}\left(x-k \frac{W \times p}{e_{x} \times(z / g)}, y-l \frac{H \times p}{e_{y} \times(z / g)}\right),
$$

where $x$ and $y$ denote the pixel coordinates of the reconstructed image, $f_{m, n}$ is the parallax image of the location of $(m, n)$ in the camera array, $g$ is the gap between reconstructed image and virtual microlens array, $e_{x} \times e_{y}$ represents the size of elemental image, and $N(x, y)$ represents the overlapping number matrix.

\section{Experiment Results and Discussion}

3.1. Experiment Results. In order to demonstrate the effectiveness of the proposed approach, we implement an experiment using two 3D objects including a Rubik's cube and a model car. The SAII pickup system consists of a camera and a motorized translation stage. The experiment setup is illustrated in Figure 5. Table 1 shows the experimental parameters.

In the experiment, we use the SAII pickup system to acquire $11 \times 11$ parallax images which record spatial and angular information of the 3D scene, and a part of parallax images are illustrated in Figure 6. For the sake of simplicity, these acquired parallax images are encrypted with the same secret keys which are generated by random number generator under the Fresnel approximation. The mapping algorithm is applied to generate the cipher image.

The decrypted parallax images are acquired by the decryption process which is a reverse operation of the encryption process. In the proposed approach, 3D information can be reconstructed flexibly according to II system configuration. The result of OIIR shows different perspectives of $3 \mathrm{D}$ scene, as shown in Figure 7. The results of the 
TABLE 1: Experimental parameters of the SAII system.

\begin{tabular}{|c|c|c|}
\hline \multirow{7}{*}{ SAII pickup parameters } & $f$-number of the camera & $F / 8.0$ \\
\hline & Focal length of the camera & $24.5 \mathrm{~mm}$ \\
\hline & Number of parallax images & $11 \times 11$ \\
\hline & Distance between two adjacent cameras & $9 \mathrm{~mm}$ \\
\hline & Distance between the camera and CDP & $1225 \mathrm{~mm}$ \\
\hline & Distance between the camera and model car & $1120 \mathrm{~mm}$ \\
\hline & Distance between the camera and Rubik's cube & $1200 \mathrm{~mm}$ \\
\hline \multirow{3}{*}{ II display parameters } & Resolution of the EIA & $3840 \times 2160$ \\
\hline & Pitch of the microlens & $1.5 \mathrm{~mm}$ \\
\hline & Focal length of the microlens & $3 \mathrm{~mm}$ \\
\hline
\end{tabular}

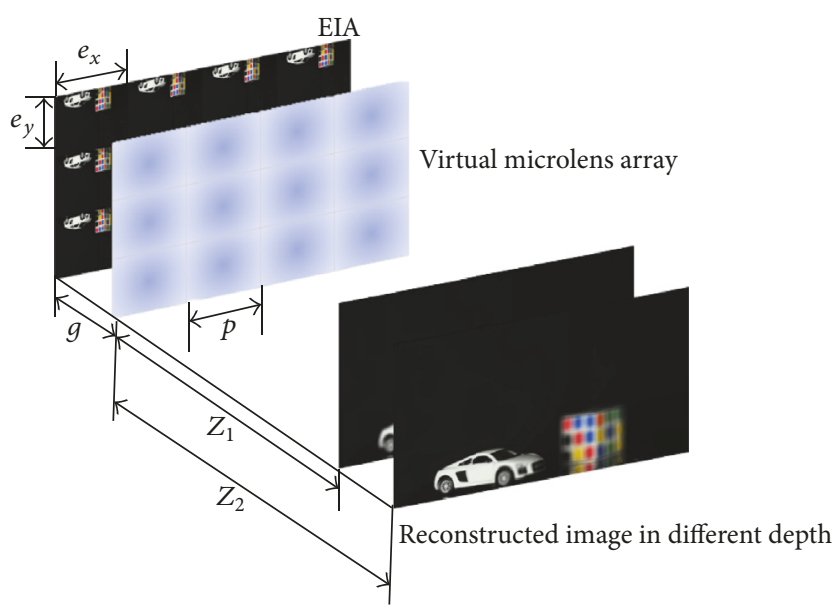

FIGURE 4: Illustration of CIIR.

reconstructed $3 \mathrm{D}$ images by CIIR which are focused on headstock, tailstock, and Rubik's cube are shown in Figure 8. It is evident that the $3 \mathrm{D}$ information can be reconstructed successfully.

\subsection{Performance Analysis}

3.2.1. Quality Analysis. Peak signal-noise ratio is applied to evaluate the decrypted image quality. The PSNR is defined as

$$
\operatorname{PSNR}(O, R)=10 \log _{10} \frac{255^{2}}{\operatorname{MSE}(x, y)},
$$

and MSE can be calculated by

$$
\operatorname{MSE}(x, y)=\frac{1}{W H} \sum_{x=0}^{W-1} \sum_{y=0}^{H-1}[O(x, y)-R(x, y)]^{2},
$$

where $O$ and $R$ represent the original image and reconstructed image, respectively, and $x$ and $y$ represent the pixel coordinates of the processed image. We calculate the average PSNR values of three channels of the reconstructed 3D images which focus on different depths, and the values are $38.25 \mathrm{~dB}, 37.58 \mathrm{~dB}$, and $38.76 \mathrm{~dB}$, respectively. The high PSNR values demonstrate the excellent quality of the proposed approach.

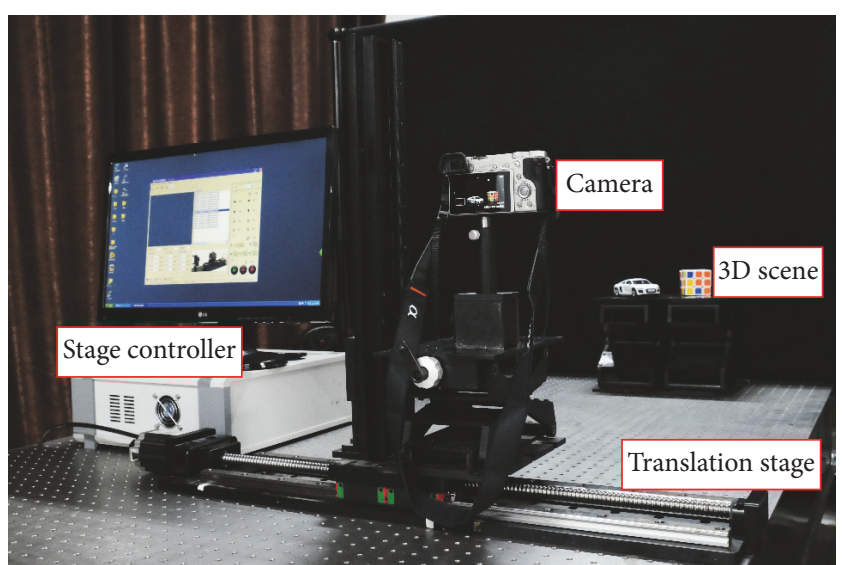

FIGURE 5: Experimental setup of the SAII pickup system.

3.2.2. Security Analysis. The sensitivity to the secret keys is an important indicator to certify the security of the proposed approach. The proposed encryption approach tremendously improves the security because of the two-step coding operation. Based on the two-step coding process, the cipher image cannot be decrypted directly by the secret keys of DRPE in Fresnel domain. To demonstrate the security of the proposed method, we simulate the case in which attackers obtain a part of the secret keys. Firstly, the first decoding is not performed, the secret keys of DRPE in Fresnel domain are used to decrypt the cipher image, and the decrypted image is shown in Figure 9(a). Secondly, the cipher image is decoded by the inverse mapping algorithm to generate a set of encrypted parallax images. Then secret keys with a wrong parameter of DRPE in Fresnel domain are used to decode one of the images; the resulting image is shown in Figure 9(b). From the results, it is very difficult for attackers to obtain $3 \mathrm{D}$ information.

Statistical information can be used to speculate the image information, and autocorrelation between the adjacent pixels can reflect the statistical information of image. We calculate the values of autocorrelation and the results are shown in Figure 10. Figures 10(a) and 10(b) show the autocorrelation values of the EIA and the cipher image, respectively. From the results, we can see that the autocorrelation of the cipher image is much weaker than EIA, which confirms that the proposed approach has high robustness against statistical attack. 


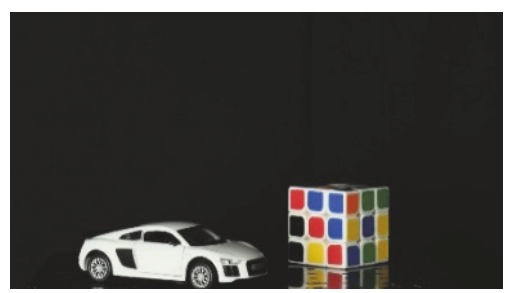

(a)

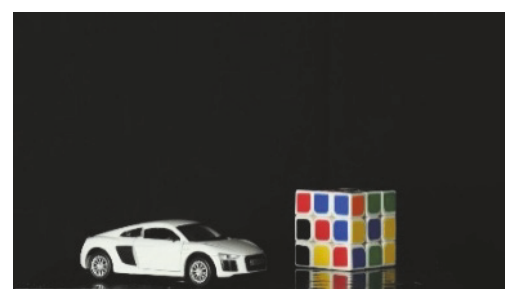

(b)

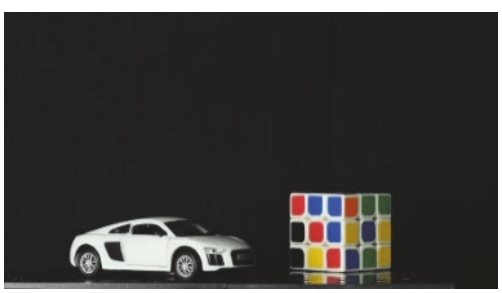

(c)

FIgURE 6: Parallax images of different perspectives: (a) $(1,1)$ th parallax image, (b) $(6,6)$ th parallax image, and $(c)(11,11)$ th parallax image.

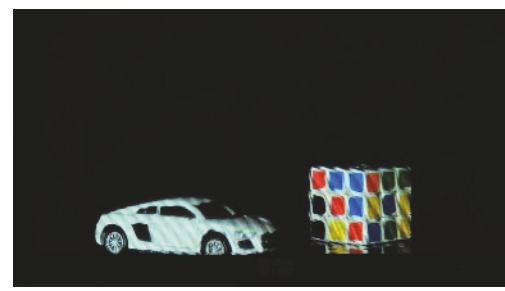

(d)

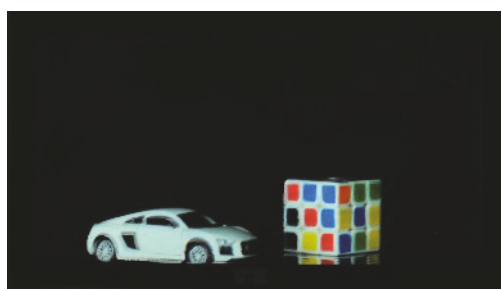

(a)

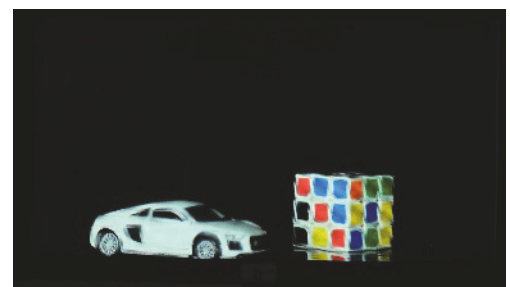

(b)

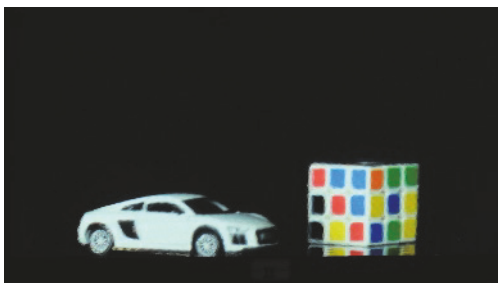

(c)

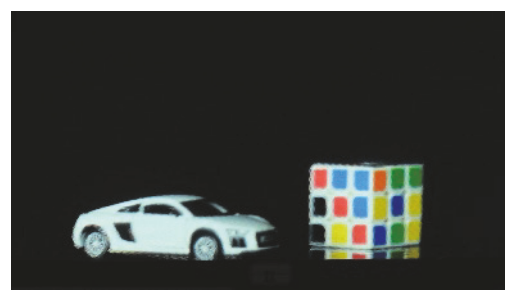

(e)

FIGURE 7: Reconstructed 3D images by OIIR with different perspectives: (a) left view, (b) front view, (c) right view, (d) top view, and (e) bottom view.

3.2.3. Robustness Analysis. To certify the robustness of the proposed encryption approach against different attacks, the Gaussian noise and occlusion attacks are adopted. We add the zero-mean Gaussian noise with different variances to the cipher image and reconstruct the corresponding 3D images by using CIIR. Figures 11(a)-11(c) show the reconstructed 3D images under the variance of 0.1 ; Figures $11(\mathrm{~d})-11(\mathrm{f})$ show the reconstructed $3 \mathrm{D}$ images under the variance of 0.2 . The values of PSNR are calculated and shown in Table 2. From the results, we can know that the $3 \mathrm{D}$ information can be clearly recognized even if the cipher image suffers from the zeromean Gaussian noise with the variance of 0.2 .

Also, the cipher image which is occluded $50 \%$ is used to test the robustness of the encryption approach against occlusion attacks. Figure 12(a) shows the vertically occluded cipher image. Correspondingly, the reconstructed 3D images focusing on different objects are obtained and shown in Figures 12(b)-12(d), respectively. The horizontally occluded cipher image is shown in Figure 12(e). The reconstructed 3D images in the different depth planes are shown in Figures 12(f)-12(h), respectively. The values of PSNR are calculated and shown in Table 3. From the reconstructed images and the calculated PSNR values, we can see that the 3D information can be reconstructed completely when the cipher image is occluded 50\%.

3.2.4. Plaintext Attack Analysis. Plaintext attacks can be implemented by attackers to obtain information, for instance, the known-plaintext attack and the chosen-plaintext attack. The known-plaintext attack is an attack method, in which the attackers can acquire original images and corresponding encrypted images. The information can be used to deduce 


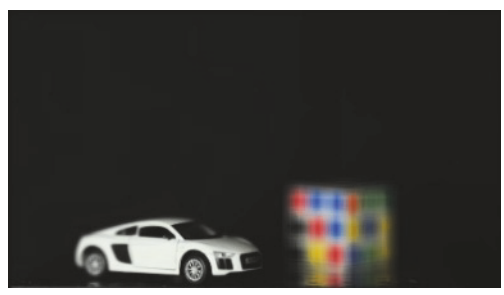

(a)

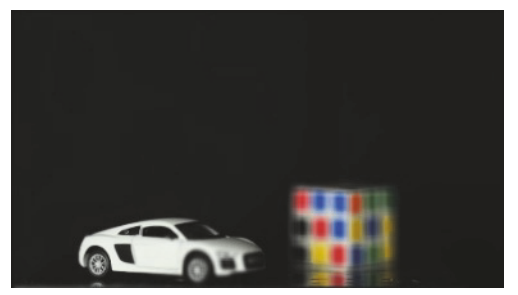

(b)

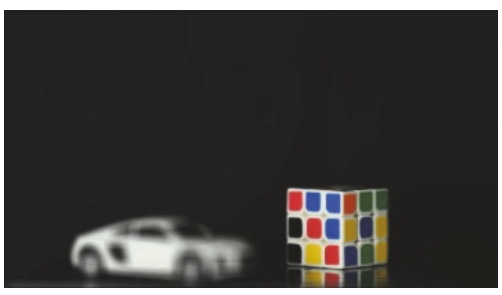

(c)

FIGURE 8: Reconstructed 3D images by CIIR with different depths: (a) headstock, (b) tailstock, and (c) Rubik's cube.

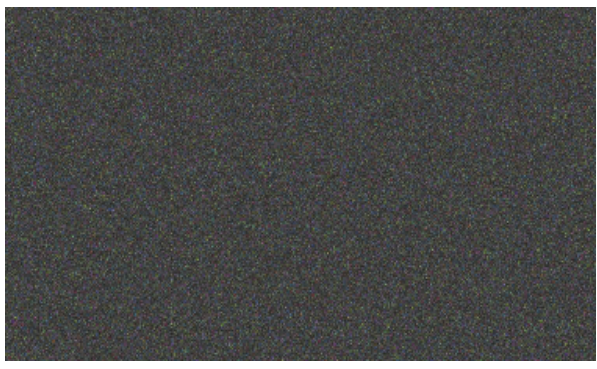

(a)

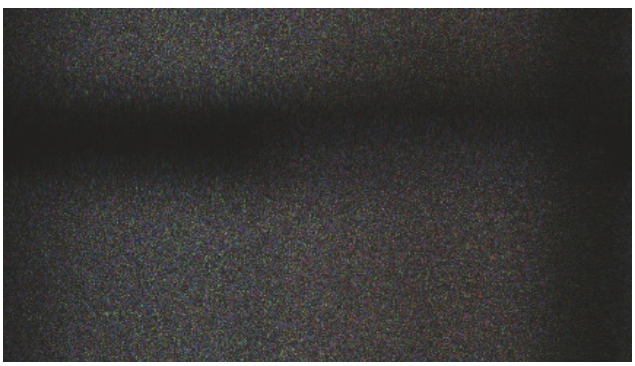

(b)

FIGURE 9: Decryption results: (a) the decrypted cipher image with correct secret keys without the first decoding and (b) the decrypted parallax image with partial incorrect keys.

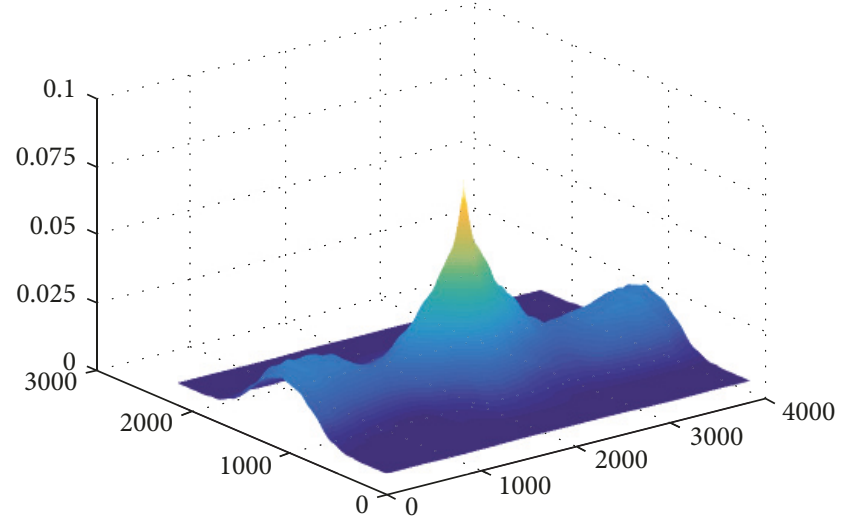

(a)

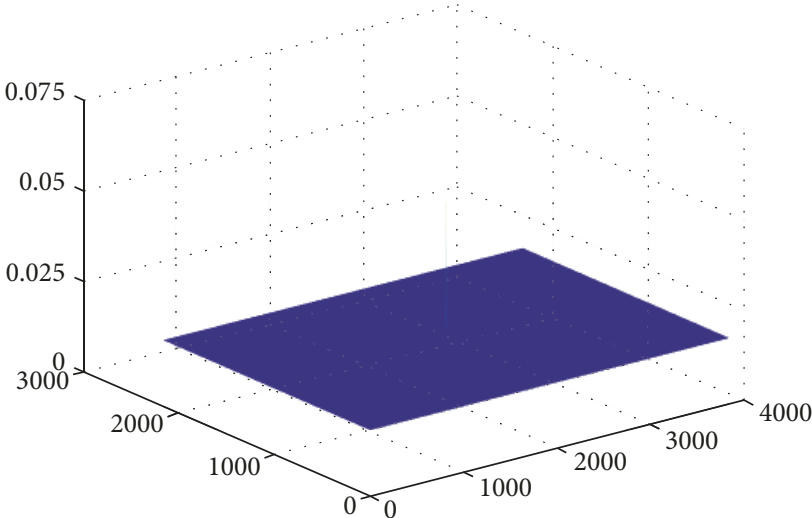

(b)

FIGURE 10: Autocorrelation results: (a) EIA and (b) the cipher image.

TABLE 2: PSNR values of CIIR from the cipher image that suffered from Gaussian noise.

\begin{tabular}{lccc}
\hline Gaussian variance & Headstock & Tailstock & Rubik's cube \\
\hline 0.1 & 19.77 & 19.64 & 19.68 \\
0.2 & 16.54 & 16.41 & 16.44 \\
\hline
\end{tabular}

TABLE 3: PSNR values of reconstructed 3D images by CIIR from the cipher image occluded 50\%.

\begin{tabular}{lccc}
\hline & Headstock & Tailstock & Rubik's cube \\
\hline Horizontally occluded $50 \%$ & 20.24 & 19.88 & 20.07 \\
Vertically occluded $50 \%$ & 20.20 & 19.89 & 20.17 \\
\hline
\end{tabular}




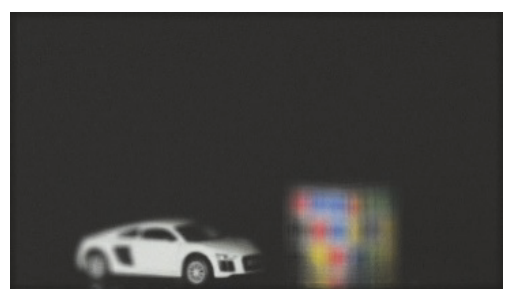

(a)

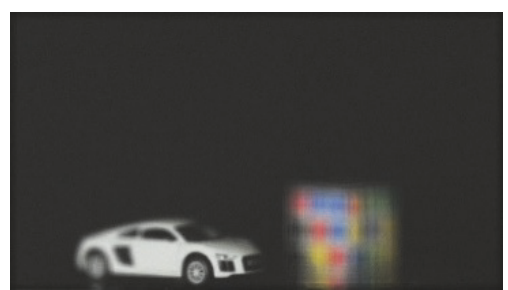

(d)

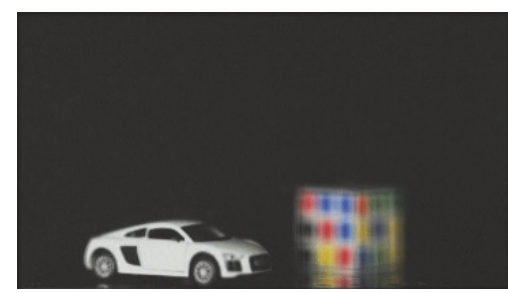

(b)

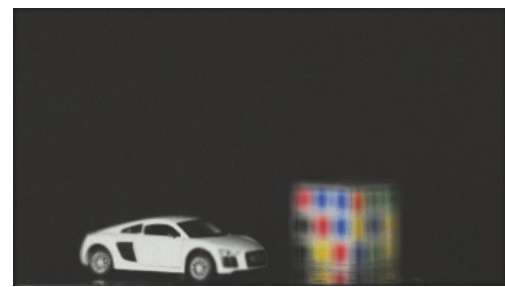

(e)

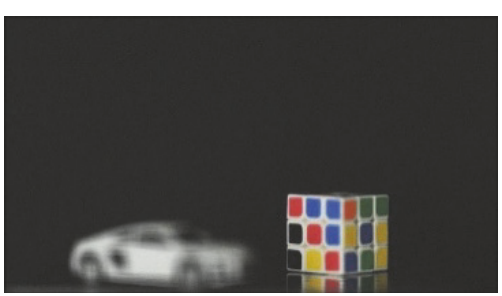

(c)

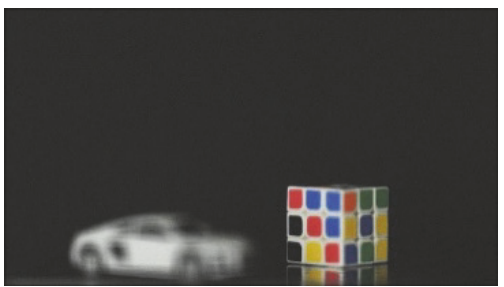

(f)

FIGURE 11: Reconstructed 3D images by CIIR from the cipher image that suffered from Gaussian noise: (a)-(c) Gaussian noise variance 0.1 and (d)-(f) Gaussian noise variance 0.2.

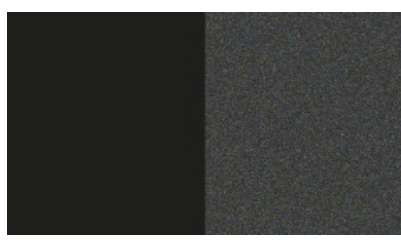

(a)

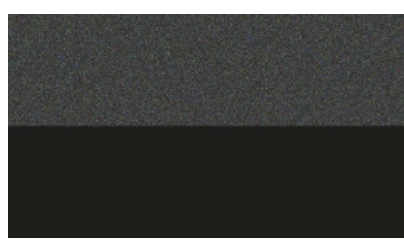

(e)

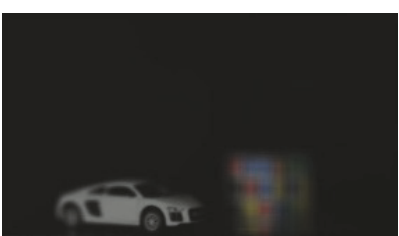

(b)

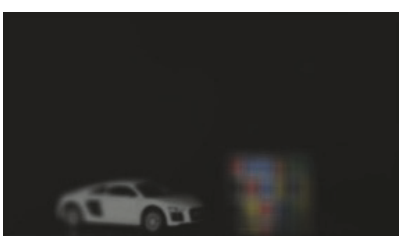

(f)

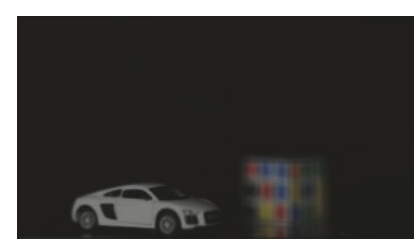

(c)

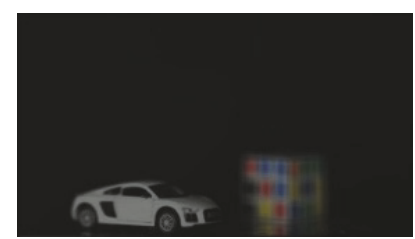

$(\mathrm{g})$

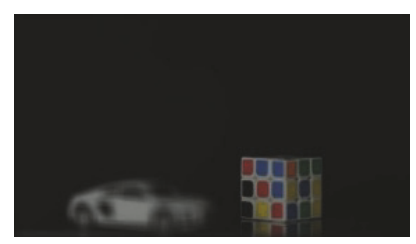

(d)

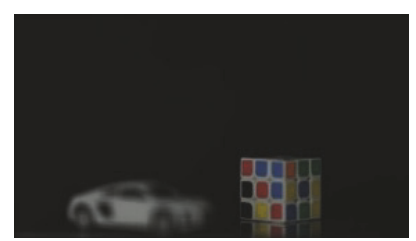

(h)

FIGURE 12: Reconstructed 3D images by CIIR from the occluded cipher image: (a) the vertical occluded cipher image, (b)-(d) different depth planes of the 3D scene reconstructed from the occluded cipher image in the vertical direction, (e) the horizontal occluded cipher image, and (f)-(h) different depth planes of the 3D scene reconstructed from the occluded cipher image in the horizontal direction.

secret keys. In the chosen-plaintext attack, it is presumed that the attackers can gain the encrypted image for each original image; therefore, the secret keys can be deduced by these pairs of original images and encrypted images.

In our two-step integral imaging coding approach, the DRPE in Fresnel domain and a mapping algorithm which is used to generate elemental image array of integral imaging are used. Each pixel of the cipher image derives from encrypted parallax images. The location of the pixel is decided by the parameters of mapping algorithm, and the value of the pixel is decided by the parameters of DRPE in Fresnel domain. The parameters of DRPE in Fresnel domain and the mapping algorithm can be both considered as secret keys. The security analysis confirms that our approach provides a high security due to big key space and two-step coding. It is very difficult for attackers that information is acquired by plaintext attacks.

\section{Conclusions}

We have presented a two-step integral imaging coding based 3D information encryption approach. In this approach, an SAII pickup system is used to transform 3D information to a set of parallax images which record different perspectives of 3D scene. DRPE in Fresnel domain is used to encrypt parallax images. The mapping algorithm is introduced to translate encrypted parallax images to a cipher image, which improves greatly the security because the cipher image cannot be decrypted straightway by decryption algorithm unless it is remapped inversely, and the parameters of the SAII pickup system can be used as the secret keys. Meanwhile, due to the property of DRPE in Fresnel domain, autocorrelation between the adjacent pixels of the cipher is much weaker, and the robustness against different attacks is higher. The 
experimental results verify the effectiveness, security, and robustness of the approach, and the $3 \mathrm{D}$ information has been reconstructed satisfactorily by OIIR and CIIR.

\section{Conflicts of Interest}

The authors declare that they have no conflicts of interest.

\section{Acknowledgments}

This work is supported by the National Defense Program of China under Grant no. 17-H863-04-ZT-001-031-01 and the NSFC under Grant nos. 61320106015 and 61705146.

\section{References}

[1] G. Lippmann, La photographie integrale, Academie des Sciences, 1908, 446-451.

[2] X. Xiao, B. Javidi, M. Martinez-Corral, and A. Stern, "Advances in three-dimensional integral imaging: Sensing, display, and applications [Invited]," Applied Optics, vol. 52, no. 4, pp. 546560,2013

[3] J.-H. Park, K. Hong, and B. Lee, "Recent progress in threedimensional information processing based on integral imaging," Applied Optics, vol. 48, no. 34, pp. H77-H94, 2009.

[4] J. Geng, "Three-dimensional display technologies," Advances in Optics and Photonics, vol. 5, no. 4, pp. 456-535, 2013.

[5] H. Navarro, R. Martínez-Cuenca, A. Molina-Martían, M. Martínez-Corral, G. Saavedra, and B. Javidi, "Method to remedy image degradations due to facet braiding in 3D integral-imaging monitors," Journal of Display Technology, vol. 6, no. 10, pp. 404-411, 2010.

[6] F. Okano, J. Arai, K. Mitani, and M. Okui, "Real-time integral imaging based on extremely high resolution video system," Proceedings of the IEEE, vol. 94, no. 3, pp. 490-500, 2006.

[7] A. Stern and B. Javidi, "Three-dimensional image sensing, visualization, and processing using integral imaging," Proceedings of the IEEE, vol. 94, no. 3, pp. 591-606, 2006.

[8] Y. Taguchi, T. Koike, K. Takahashi et al., "TransCAIP: A live 3D TV system using a camera array and an integral photography display with interactive control of viewing parameters," IEEE Transactions on Visualization \& Computer Graphics, vol. 15, no. 5, pp. 841-852, 2009.

[9] J.-S. Jang and B. Javidi, "Three-dimensional synthetic aperture integral imaging," Optics Expresss, vol. 27, no. 13, pp. 1144-1146, 2002.

[10] M. Levoy, "Light fields and computational imaging," The Computer Journal, vol. 39, no. 8, pp. 46-55, 2006.

[11] S.-H. Hong, J.-S. Jang, and B. Javidi, “Three-dimensional volumetric object reconstruction using computational integral imaging," Optics Express, vol. 12, no. 3, pp. 483-491, 2004.

[12] H. Arimoto and B. Javidi, "Integral three-dimensional imaging with digital reconstruction," Optics Expresss, vol. 26, no. 3, pp. 157-159, 2001.

[13] W. Chen, B. Javidi, and X. Chen, "Advances in optical security systems," Advances in Optics and Photonics, vol. 6, no. 2, pp. 120155, 2014.

[14] N.-R. Zhou, Y. Wang, and L. Gong, "Novel optical image encryption scheme based on fractional Mellin transform," Optics Communications, vol. 284, no. 13, pp. 3234-3242, 2011.
[15] W. Chen, X. Chen, and C. J. R. Sheppard, “Optical double-image cryptography based on diffractive imaging with a laterallytranslated phase grating," Applied Optics, vol. 50, no. 29, pp. 5750-5757, 2011.

[16] S. You, Y. Lu, W. Zhang, B. Yang, R. Peng, and S. Zhuang, "Micro-lens array based 3-D color image encryption using the combination of gravity model and Arnold transform," Optics Communications, vol. 355, pp. 419-426, 2015.

[17] Z. Liu, C. Shen, J. Tan, and S. Liu, "A recovery method of double random phase encoding system with a parallel phase retrieval," IEEE Photonics Journal, vol. 8, no. 1, 2016.

[18] N. Zhou, T. Dong, and J. Wu, "Novel image encryption algorithm based on multiple-parameter discrete fractional random transform," Optics Communications, vol. 283, no. 15, pp. 3037$3042,2010$.

[19] N. Zhou, S. Pan, S. Cheng, and Z. Zhou, "Image compressionencryption scheme based on hyper-chaotic system and 2D compressive sensing," Optics \& Laser Technology, vol. 82, pp. 121-133, 2016.

[20] M. R. Abuturab, "An asymmetric single-channel color image encryption based on Hartley transform and gyrator transform," Optics and Lasers in Engineering, vol. 69, pp. 49-57, 2015.

[21] Z. Hua and Y. Zhou, "Design of image cipher using block-based scrambling and image filtering," Information Sciences, vol. 396, pp. 97-113, 2017.

[22] X. W. Li, D. Xiao, and Q. H. Wang, "Error-free holographic frames encryption with CA pixel-permutation encoding algorithm," Optics and Lasers in Engineering, vol. 100, pp. 200-207, 2018.

[23] X. Li, C. Li, and I.-K. Lee, "Chaotic image encryption using pseudo-random masks and pixel mapping," Signal Processing, vol. 125, pp. 48-63, 2016.

[24] N. Zhou, Y. Wang, L. Gong, H. He, and J. Wu, "Novel singlechannel color image encryption algorithm based on chaos and fractional Fourier transform," Optics Communications, vol. 284, no. 12, pp. 2789-2796, 2011.

[25] N. Zhou, H. Li, D. Wang, S. Pan, and Z. Zhou, "Image compression and encryption scheme based on 2D compressive sensing and fractional Mellin transform," Optics Communications, vol. 343, pp. 10-21, 2015.

[26] G. H. Situ and J. J. Zhang, "Double random-phase encoding in the Fresnel domain," Optics Expresss, vol. 29, no. 14, pp. 15841586,2004

[27] M. Cho and B. Javidi, "Three-dimensional photon counting double-random-phase encryption," Optics Expresss, vol. 38, no. 17, pp. 3198-3201, 2013.

[28] I. Muniraj, C. Guo, B.-G. Lee, and J. T. Sheridan, "Interferometry based multispectral photonlimited 2D and 3D integral image encryption employing the Hartley transform," Optics Express, vol. 23, no. 12, pp. 15907-15920, 2015.

[29] D. H. Kim, Y. R. Piao, S. J. Cho et al., "3D Image Encryption Using Integral Imaging Scheme and MLCA Technology," Applied Mechanics \& Materials, vol. 284-287, no. 1, pp. 29552960, 2013

[30] Y. R. Piao, D. H. Shin, and E. S Kim, "Robust image encryption by combined use of integral imaging and pixel scrambling techniques," Optics \& Lasers in Engineering, vol. 47, no. 11, pp. 1273-1281, 2009

[31] X. W. Li and I. K. Lee, "Modified computational integral imaging-based double image encryption using fractional Fourier transform," Optics \& Lasers in Engineering, vol. 66, no. 9, pp. 112-121, 2015. 
[32] Y. Xing, Q.-H. Wang, Z.-L. Xiong, and H. Deng, "Encrypting three-dimensional information system based on integral imaging and multiple chaotic maps," Optical Engineering, vol. 55, no. 2, Article ID 023107, 2016. 


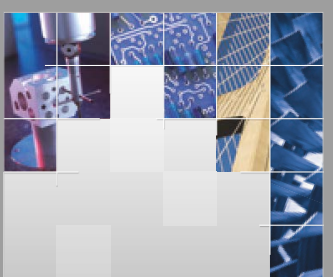

\section{Enfincering}
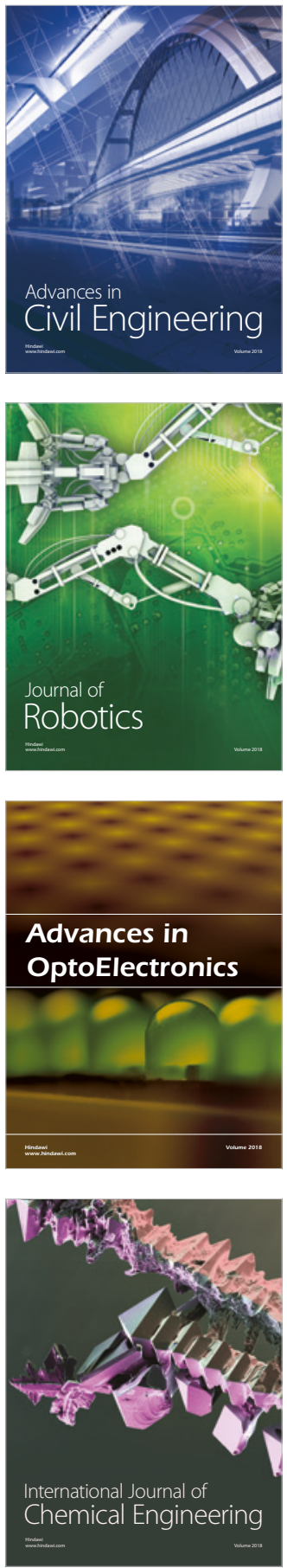

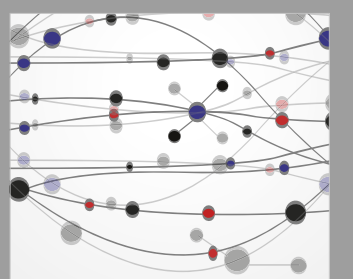

\section{Rotating \\ Machinery}

The Scientific World Journal

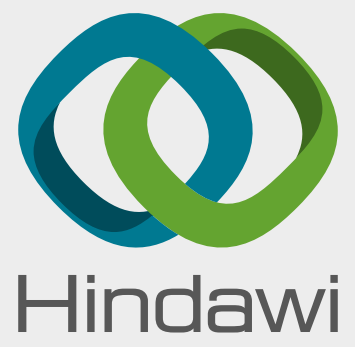

Submit your manuscripts at

www.hindawi.com
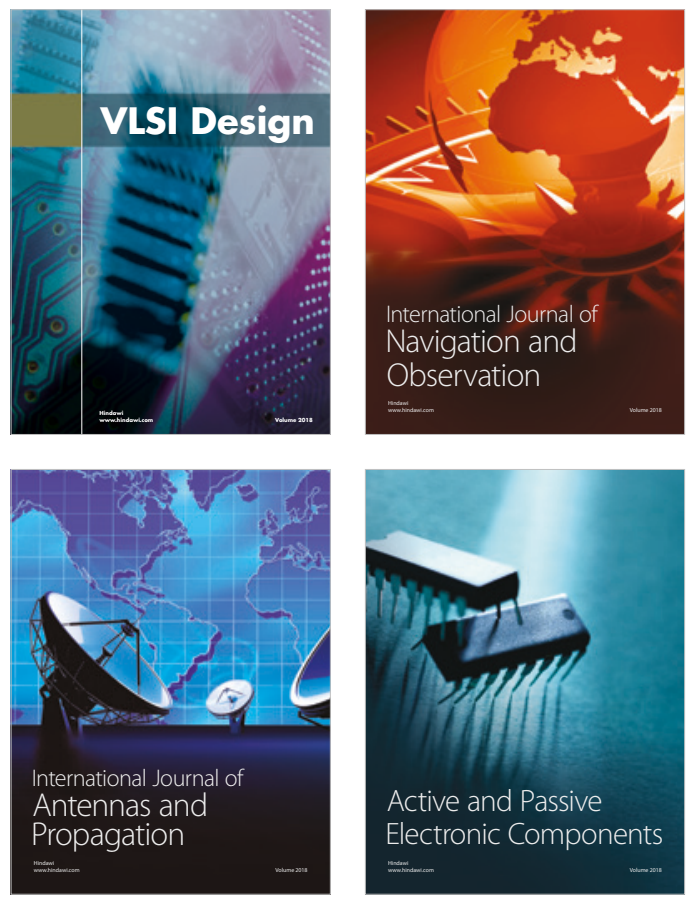
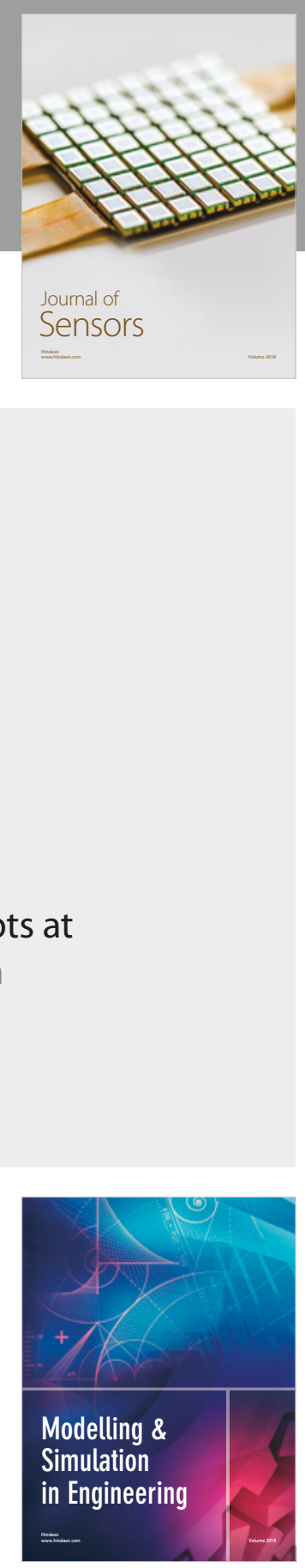

\section{Advances \\ Multimedia}
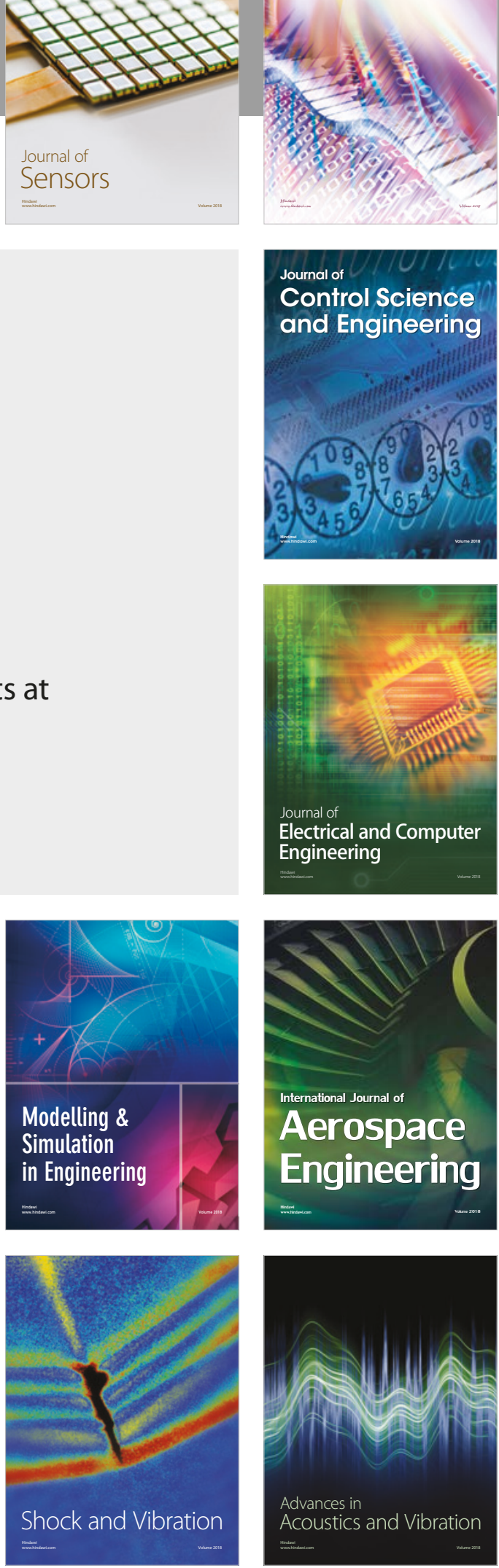